\title{
Acute-onset rhabdomyolysis secondary to sitagliptin and atorvastatin interaction
}

This article was published in the following Dove Press journal:

International Journal of General Medicine

29 April 2016

Number of times this article has been viewed

\section{Muhammad Waqas Khan'}

Saji Kurian²

Rohit Bishnoi'

'Division of Hospital Medicine, Department of Medicine, College of Medicine, ${ }^{2}$ College of Pharmacy, University of Florida, Gainesville, FL, USA
Correspondence: Rohit Bishnoi Division of Hospital Medicine, Department of Medicine, College of Medicine, University of Florida, Gainesville, FL 32610, USA

Tel +l 3525943589

Fax +I 3522650379

Email Rohit.Bishnoi@medicine.ufl.edu

\begin{abstract}
Rhabdomyolysis is a serious medical condition in which the skeletal muscle tissue gets damaged and breaks down at rapid rates, potentially leading to death if not managed early on. Rhabdomyolysis in adults has several etiologies such as crush injuries, prolonged immobilization, strenuous exercise, hormonal or metabolic causes, infections, and drug-drug interactions. We present a case report of the interaction of two drugs that are used commonly in the general population. We here discuss a case of a 60 -year-old female who presented to the hospital with complaints of generalized weakness, muscle aches, and atypical chest pain for a week after her primary care physician started her on sitagliptin while she was already on atorvastatin. After review of literature, this is the second known case of such an interaction causing acute breakdown of skeletal musculature.
\end{abstract}

Keywords: rhabdomyolysis, drug interaction, statin-sitagliptin interaction

\section{Introduction}

With the incidence of obesity on the rise, an increasing number of patients are being frequently diagnosed with complications secondary to their high body mass index. Diabetes mellitus (DM), hyperlipidemia, and coronary artery disease are a few of the more common diseases that seem to affect such individuals. Although several studies have been conducted to evaluate the safety profiles and interactions among the drug groups used to treat and manage these illnesses, unusual side effects may still arise in some patients. ${ }^{1-5}$ We aim to create awareness regarding one of the frequently used combinations of drugs, namely, sitagliptin and atorvastatin, the use of which resulted in rhabdomyolysis of the skeletal muscle in a patient. Although an isolated case, we propose and encourage further clinical trials in this area.

\section{Case report}

A 60-year-old female with past medical history significant for hypertension, DM, coronary artery disease, and hyperlipidemia presented to the emergency room with the chief complaint of chest pain. She also reported generalized weakness, myalgias, subjective fever, and chills progressively worsening over the past 7 days. Her physical examination showed stable vital signs and was otherwise unremarkable except for trace peripheral edema. The University of Florida does not require IRB approval or written informed consent for any case report with 3 or fewer patients as it is not a clinical study.

Fearing a coronary event, she was given a dose of sublingual nitroglycerine and aspirin. An electrocardiogram showed sinus tachycardia, but otherwise no apparent 
abnormality. Laboratory workup was significant for elevated creatinine phosphokinase (CPK) of 13,456 U/L (reference range: $30-170 \mathrm{U} / \mathrm{L}$ ), normal troponin-T, normal complete blood count, and basic metabolic panel except elevated creatinine of $1.20 \mathrm{mg} / \mathrm{dL}$ (reference range: $0.40-0.90 \mathrm{mg}$ / dL). Her chest X-ray revealed no abnormality. CPK levels evaluated in 2012 on this patient while on statins were unremarkable (191 U/L, $165 \mathrm{U} / \mathrm{L}$, and $197 \mathrm{U} / \mathrm{L}$, with reference range of 30-170 U/L).

She was admitted to the hospital due to concern for acute coronary event, rhabdomyolysis, acute renal insufficiency, and possible acute viral infection causing her symptoms. Her workup during hospital stay showed three normal serial troponin- $\mathrm{T}$ tests, and myocardial perfusion test revealed no abnormality. Her respiratory viral panel and thyroid stimulation hormone level were normal. Her creatinine also normalized with intravenous (IV) fluids. She had persistent elevated CPK levels, which also trended down with IV fluids.

Common differential diagnoses for her rhabdomyolysis were explored by detailed history and laboratory workup. There was no history of recent trauma, crush injury, prolonged immobilization, recent surgery, seizures, drug abuse, or alcoholism. Her family history was negative for any kind of myopathy or other hereditary or genetic causes that could explain the elevated CPK value. Her laboratory workup, including thyroid function tests, was unremarkable and failed to explain this episode of rhabdomyolysis. She had been on a stable dose of atorvastatin (40 mg) for several years for hyperlipidemia and reported no myalgias, arthralgias, or abnormal CPK levels. Review of her medication list further revealed that she was started on sitagliptin (Januvia ${ }^{\circledR} 100 \mathrm{mg}$ ) almost a week before this presentation. She also correlated her symptoms with starting of this new medication. Sitagliptin and atorvastatin were stopped; her subsequent CPK value showed a decreasing trend and was recorded at 1,220 U/L on the day of discharge. The patient's muscle aches and chest pain had reduced considerably following the discontinuation of these medications.

\section{Discussion}

Rhabdomyolysis is potentially a serious clinical condition that involves breakdown of muscle tissue, releasing myoglobin into the blood. It can be caused by injuries to the skeletal muscles, which include trauma or crush injuries, severe exertion, dehydration, genetic muscle diseases, elevated body temperature, muscle tissue ischemia, electrolyte imbalance, and seizures, and can sometimes result from drug interactions. A few signs and symptoms of rhabdomyolysis include body aches, generalized weakness, myalgia, fever, and sometimes hematuria.

Atorvastatin belongs to the family of inhibitors of 3-hydroxy-3-methylglutaryl coenzyme A (HMG-CoA) reductase, frequently known as statins, responsible for the conversion of HMG-CoA to mevalonate, preventing the cholesterol synthesis pathway. It is metabolized to its active metabolites, ortho- and para-hydroxylated forms, which are responsible for a majority of reactions. Atorvastatin is a substrate for hepatic cytochrome P450 (CYP) 3A4, as well as $\mathrm{P}$-glycoprotein and the transporter organic anion-transporting polypeptide 1B1 (OATP1B1). ${ }^{6-8}$ This in turn tends to cause significant drug interactions with other CYP3A4 inhibitors, which may result in potential complications such as myopathies.

Sitagliptin belongs to a newer class of agents known as dipeptidyl peptidase 4 (DPP-IV) inhibitors approved in 2006 by the US Food and Drug Administration (FDA) for the treatment of type 2 DM. DPP-IV inhibitors slow down the inactivation of incretin hormones (glucagon-like peptide-1 [GLP-1] and glucose-dependent insulinotropic polypeptide [GIP]). These hormones control blood glucose by increasing synthesis and release of insulin from pancreatic $\beta$ cells in response to a meal. GLP-1 also reduces hepatic gluconeogenesis by inhibiting the pancreatic $\alpha$ cells. ${ }^{9}$ Sitagliptin is primarily excreted in the urine as the unchanged drug by renal tubular secretion. About $16 \%$ of the drug is excreted as its metabolites and primarily metabolized by CYP3A4, CYP2C8, and P-glycoprotein. Sitagliptin is not an inhibitor of CYP isozymes 3A4, 2C8, 2C9, 2D6, 1A2, 2C19, or 2B6. It is neither an inducer of CYP3A4 nor does it inhibit P-glycoprotein-mediated transport. ${ }^{10}$

A study by Wilke et $\mathrm{al}^{11}$ showed that a nonsynonymous coding single-nucleotide polymorphism - rs4149056 - in SLCO1B1 significantly increased the exposure to simvastatin and the risk of muscle toxicity. OATP1B1, encoded by $S L C O 1 B 1$, facilitates the hepatic uptake of several endogenous compounds and statins. Changes in the activity of this transporter can increase the severity of statinrelated muscle damage. Genetic variability in SLCO1B1 also alters plasma concentration of statins, although the overall pharmacokinetic profile of simvastatin appears to be altered more than that of any other drug in the class. ${ }^{12,13}$ Pasanen et $\mathrm{al}^{14}$ demonstrated that homozygous carriers of the C allele at rs4149056 had a greater exposure to the active simvastatin acid than subjects homozygous for the ancestral $\mathrm{T}$ allele. 
The frequency of mylagias with atorvastatin was $2.7 \%-8.4 \%$ vs $3.1 \%$ in patients receiving placebo and is one of the common reported skeletal muscle side effects seen with the use of atorvastatin $\left(\right.$ Lipitor $\left.^{\mathbb{8}}\right) .{ }^{8}$ In contrast, a study by Graham et $\mathrm{al}^{9}$ in 2004 reported an average incidence of 0.44 in 10,000 patients (who developed rhabdomyolysis) being treated with atorvastatin, simvastatin, or pravastatin, with a $95 \%$ confidence interval of $0.20-0.84$. These patients had been taking lipidlowering agents alone or in combination. ${ }^{9}$ The FDA reported a total of 601 cases of statin-associated rhabdomyolysis from November 1997 up till March 2000. ${ }^{15}$ The total case percentages associated with each statin were as follows: fluvastatin at $2 \%$, lovastatin at $7 \%$, pravastatin at $12 \%$, atorvastatin at $12 \%$, cerivastatin at $32 \%$, and simvastatin at $36 \%$. In a similar study by Thompson et al, ${ }^{16}$ an expanded search was carried out to ensure compatibility with prior searches and 612 cases were identified for the same given period. The incidence of fatal rhabdomyolysis through May in 2001 had been estimated using information from the databases obtained from the FDA and National Prescription Audit Plus (IMS Health, Fairfield, CT, USA) and found to be at only 0.15 deaths per one million prescriptions. ${ }^{17}$ The estimated incidences per one million prescriptions for various statins were as follows: cerivastatin at 3.16 , lovastatin at 0.19 , simvastatin at 0.12 , pravastatin and atorvastatin at 0.04 , and fluvastatin at zero. ${ }^{17}$

Diltiazem is considered a weak inhibitor of the CYP3A4 isoenzyme and although our patient had been taking diltiazem (180 mg daily) along with atorvastatin for several months, it would be an unlikely cause for this acute rhabdomyolysis episode.

The patient developed rhabdomyolysis within a few days after sitagliptin was added to her continuing medical therapy, which included atorvastatin. Both atorvastatin and sitagliptin are substrates for CYP3A4 and P-glycoprotein. She did not have any other known etiologies for developing rhabdomyolysis, leading to the conclusion that the drug interaction between atorvastatin and sitagliptin caused toxicity and rhabdomyolysis.

Medical literature review showed very limited case reports of potential interaction of sitagliptin with statins causing rhabdomyolysis. ${ }^{518}$ Although sitagliptin has been reported to cause myalgias or arthralgias, it has not been reported to cause rhabdomyolysis on its own., ${ }^{5,18-19}$

\section{Conclusion}

Several drug interactions have been reported with statins in the past. To our knowledge, however, only one case has been reported on the interaction of sitagliptin with statins causing rhabdomyolysis, making this case an unusual presentation. A medication review should thoroughly be done in case of myalgias in the absence of any medical or traumatic event likely to cause muscle damage.

\section{Disclosure}

The authors report no conflicts of interest in this work. The authors have not received funding from any organization.

\section{References}

1. Thompson PD, Clarkson PM, Rosenson RS, National Lipid Association Statin Safety Task Force Muscle Safety Expert Panel. An assessment of statin safety by muscle experts. Am J Cardiol. 2006; 97(8A):69C-76C.

2. Armitage J, Bowman L, Wallendszus K, et al. Study of the Effectiveness of Additional Reductions in Cholesterol and Homocysteine (SEARCH) Collaborative Group. Lancet. 2010; 376(9753):1658-69.

3. US Food and Drug Adminsitration. FDA Drug Safety Communication: New restrictions, contraindications, and dose limitations for Zocor (simvastatin) to reduce the risk of muscle injury. Avaialble from http:// www.fda.gov/Drugs/DrugSafety/ucm283137.htm. Accessed Apr 4, 2016.

4. Kostapanos MS, Milionis HJ, Elisaf MS. Rosuvastatin-associated adverse effects and drug-drug interactions in the clinical setting of dyslipidemia. Am J Cardiovasc Drugs. 2010;10(1):11-28.

5. Bhome R, Penn H. Rhabdomyolysis precipitated by a sitagliptinatorvastatin drug interaction. Diabet Med. 2012;29:693-694.

6. Williams D, Feely J. Pharmacokinetic-pharmacodynamic drug interactions with HMG-CoA reductase inhibitors. Clin Pharmacokinet. 2002;41:343-370.

7. Lau YY, Huang Y, Frassetto L, Benet LZ. Effect of OATP1B transporter inhibition on the pharmacokinetics of atorvastatin in healthy volunteers. Clin Pharmacol Ther. 2007;81:194-201.

8. Lipitor (atorvastatin calcium) [package insert]. New York, NY: ParkeDavis; 2015.

9. Graham DJ, Staffa JA, Shatin D, et al. Incidence of hospitalized rhabdomyolysis in patients treated with lipid-lowering drugs. JAMA. 2004;292(21):2585-2590.

10. Sitagliptin (Januvia) [package insert]. Whitehouse station, NJ: Merk and Co Inc.; 2015.

11. Wilke RA, Ramsey LB, Johnson SG, et al. The clinical pharmacogenomics implementation consortium: CPIC guideline for SLCO1B1 and simvastatin-induced myopathy. Clin Pharmacol Ther. 2012;92(1):112-117.

12. Niemi M, Pasanen MK, Neuvonen PJ. Organic anion transporting polypeptide $1 \mathrm{~B} 1$ : a genetically polymorphic transporter of major importance for hepatic drug uptake. Pharmacol Rev. 2011;63(1):157-181.

13. Kalliokoski A, Niemi M. Impact of OATP transporters on pharmacokinetics. Br J Pharmacol. 2009;158(3):693-705.

14. Pasanen MK, Neuvonen M, Neuvonen PJ, Niemi M. SLCO1B1 polymorphism markedly affects the pharmacokinetics of simvastatin acid. Pharmacogenet Genomics. 2006;16(12):873-879.

15. Omar MA, Wilson JP. FDA adverse event reports on statin-associated rhabdomyolysis. Ann Pharmacother. 2002;36:288-295.

16. Thompson PD, Clarkson P, Karas RH. Statin-associated myopathy. JAMA. 2003;289(13):1681-1690.

17. Staffa JA, Chang J, Green L. Cerivastatin and reports of fatal rhabdomyolysis. N Engl J Med. 2002;346:539-540.

18. DiGregorio RV, Pasikhova Y. Rhabdomyolysis caused by a potential sitagliptin-lovastatin interaction. Pharmacotherapy. 2009;29(3): 352-356.

19. Kao DP, Kohrt HE, Kugler J. Renal failure and rhabdomyolysis associated with sitagliptin and simvastatin use. Diabet Med. 2008; 25(10):1229-1230. 


\section{Publish your work in this journal}

The International Journal of General Medicine is an international, peer-reviewed open-access journal that focuses on general and internal medicine, pathogenesis, epidemiology, diagnosis, monitoring and treatment protocols. The journal is characterized by the rapid reporting of reviews, original research and clinical studies across all disease areas.

A key focus is the elucidation of disease processes and management protocols resulting in improved outcomes for the patient. The manuscript management system is completely online and includes a very quick and fair peer-review system. Visit http://www.dovepress.com/ testimonials.php to read real quotes from published authors.

Submit your manuscript here: http://www.dovepress.com/international-journal-of-general-medicine-journal 